\title{
Rodolfo Lenz y la enseñanza del castellano como idioma patrio en Chile
}

\author{
Guillermo Soto* \\ Universidad de Chile, Chile
}

\begin{abstract}
Resumen
Algo menos conocida que sus investigaciones lingüísticas y antropológicas es la labor que Rodolfo Lenz (1863-1938) desarrolló en relación con la enseñanza del castellano como idioma patrio en Chile. Dos fueron los problemas fundamentales que abordó a este respecto, ambos de gran importancia para el diseño curricular y los programas de la asignatura de castellano. En primer término, la cuestión de qué "lengua literaria" se enseñaría, esto es, en el lenguaje actual, qué propiedades debería tener la variedad estándar de la escolarización. En segundo lugar, el problema de cómo se realizaría la instrucción en los liceos y, particularmente, del papel de la gramática en este proceso, que consideró secundario respecto de la práctica del idioma. En ambos casos, sus planteamientos se apartaron de la tradición bellista. Junto con fundamentar teórica y empíricamente sus opciones, Lenz difundió sus ideas en la opinión pública de modo de incidir políticamente en el diseño curricular y en la formación de los futuros profesores de castellano, procurando también reformar la enseñanza gramatical en Chile. Todas estas acciones las desarrolló
\end{abstract}

\footnotetext{
* Para correspondencia, dirigirse a: Guillermo Soto (gsoto@u.uchile.cl), Departamento de Lingüística, Facultad de Filosofía y Humanidades, Universidad de Chile, Av. Capitán Ignacio Carrera Pinto 1025, 3er piso, Nuñoa, CP 7800284, Santiago, Chile.
} 
en un ambiente ideológico que no era, en principio, favorable a ideas críticas de las doctrinas de Bello.

Palabras clave: Rodolfo Lenz, lingüística educacional, historia de las ideas lingüísticas, lengua estándar.

\section{Rudolf Lenz and the teaching of Spanish as a First language IN CHILE}

Abstract

Less known than his linguistic and anthropological research is the work developed by Rudolph Lenz (1863-1938) in relation to the teaching of Spanish as a first language in Chilean educational system. He focused on two fundamental problems of great importance for curriculum and program design. First, the question of what "literary language" would be taught, that is, in today's terms, which properties should belong to the standard language. Second, the problem of how instruction should be conducted in high schools, and, particularly, the role of grammar in this process. In both cases, Lenz departed from the tradition of Bello's ideas. In addition to theoretically and empirically substantiate his proposals, Lenz spread his ideas in public opinion to influence curriculum design and training of future teachers of Spanish. He also sought to reform the teaching of grammar in Chile. All this was done in an environment that was not conducive to ideas that criticized the doctrines of Bello.

Keywords: Rudolf Lenz, Educational Linguistics, History of Linguistic Ideas, Standard Language.

Recibido: $15 / 03 / 16 \quad$ Aceptado: $10 / 05 / 16$

\section{INTRODUCCIÓN}

El estudio de las ideas lingüísticas en Chile y su relación con las políticas idiomáticas se ha concentrado, preferentemente, en el período que abarca, en términos gruesos, la denominada república autoritaria del siglo XIX. Como se sabe, durante estos años la cuestión lingüística, y, más específicamente, el problema de la manera en que la República se apropiaría de la lengua española, estuvo en el centro de la discusión intelectual. Las figuras de 
Sarmiento y Bello se constituyeron en dos polos simbólicos del continuo de actitudes que mantendrían las elites de los estados que habían alcanzado recientemente la independencia (v., Gallardo 1982, Guitarte 1991, Blanco 1994). De un lado, el impulso, expreso o implícito, de una ruptura con lo que se consideraba la lengua de España, conducta que conduciría, más tarde, al intento de desarrollar lenguas nacionales o "idiomas patrios". Del otro, la preocupación por mantener la unidad lingüística panhispánica a través de "la conservación de la lengua de nuestros padres en su posible pureza" (Bello 1847 [1928]: vii).

Lejos de tratarse de una disputa puramente académica, las posturas tenían que ver tanto con la construcción de la identidad nacional por parte de las repúblicas hispanoamericanas, como con la determinación de las políticas lingüísticas y, más ampliamente, culturales e institucionales que se desarrollarían en ellas. El empleo de tal o cual convención ortográfica, la aceptación de americanismos, el nombre que llevaría la lengua no eran, simplemente, cuestiones científicas o de conveniencia práctica; constituían, más bien, puntas de lanza del tipo de sociedad que se quería construir. En este sentido, las discusiones lingüísticas se integraban en el proceso arquitectónico republicano que, dirigido por el Estado, siguió a las fases agonales de la independencia y la anarquía ${ }^{1}$.

La figura de Bello resulta paradigmática de esta unión entre el pensamiento lingüístico y las políticas públicas ${ }^{2}$. En él confluyeron no solo el gramático y el publicista preocupado de enmendar los "vicios idiomáticos" a través de columnas de prensa. Bello también fue redactor del Código Civil; primer rector de la Universidad de Chile, instaurada en 1842; senador vitalicio de la República y formador de la elite intelectual del país, integrada por miembros de la clase dirigente nacional: la aristocracia criolla. No es extraño, en consecuencia, que las ideas lingüísticas de Bello fundamentaran, de manera más o menos directa, las políticas de planificación lingüística implementadas durante los gobiernos conservadores y gran parte de los liberales.

En contraste con el período de Bello y Sarmiento, parecen más escasos los estudios sobre la época que cubre, en Chile, desde fines del siglo XIX hasta

\footnotetext{
1 La idea de que el Estado chileno construyó la nación, y no al revés, ha sido propuesta por Góngora (1981). El concepto mismo de nación parece ser un constructo histórico en cuya caracterización y construcción social han participado diversos factores, entre otros, el papel desempeñado por los Estados (v. Hobsbawm 1991).

2 El caso de Bello no constituye una singularidad. Como afirma Daoust (1997): "Linguistic change is often brought about by individuals and organizations who exert pressure on governments, embodying the aspirations of their speech community" (p. 447).
} 
las primeras décadas del siglo XX. Se trata éste de un período de especial relevancia en lo que respecta a la relación entre las ideas lingüísticas y las políticas idiomáticas. En efecto, en el contexto de un proceso más amplio de "institucionalización, expansión y modernización de la educación laica" (Subercaseaux 1997: 142), se funda, en esa época, el Instituto Pedagógico de la Universidad de Chile (1889), destinado a la formación de los profesores de educación secundaria, entre éstos, los de "idioma patrio". Junto a ello, se reforma la enseñanza del "castellano" 3 y de las lenguas extranjeras, reemplazándose la instrucción tradicional, centrada, en un caso, en la gramática, y en el otro, en la traducción, por el denominado "método directo", que pretendía replicar las condiciones de adquisición de la lengua materna coloquial por el niño.

En este mismo marco, se da también un cambio de actitud con respecto a la propia Gramática de Bello, algunos de cuyos planteamientos comienzan a ser criticados hasta llegar a sustituirse, en la enseñanza primaria y secundaria, la terminología gramatical de Bello por la académica. Se inician, además, los estudios científicos del español de Chile como variedad dialectal de la lengua española, lo que tiene por efecto la descripción de sus propiedades, con especial atención a las peculiaridades fonéticas. Junto a lo anterior, se propone una hipótesis de alcance general sobre el origen de las propiedades del español de Chile, y, por extensión, de América: la hipótesis indigenista. Aparecen, también, trabajos lexicográficos modernos enmarcados en un proyecto mayor de describir el vocabulario empleado en América. Comienza la investigación científica de la lengua mapuche, bajo el supuesto de que su estudio permitiría una mejor comprensión del español hablado en Chile. Continúa la disputa sobre la reforma ortográfica, que se resuelve definitivamente solo en la tercera década del siglo XX. Y se instaura, finalmente, la lingüística como disciplina científica en el país.

De manera similar a lo que había ocurrido con Bello en el período anterior -aunque sin la influencia totalizadora en el ámbito cultural que alcanzó el pensador venezolano-, en todas las transformaciones a que nos hemos referido tuvo participación principal el lingüista alemán Rodolfo Lenz (1863-1938): sus ideas lingüísticas constituyeron un sistema que guió una serie de cambios en el campo de la enseñanza del idioma patrio y los estudios sobre el lenguaje, además de otros dominios que escapan al objeto de esta investigación.

\footnotetext{
3 No se discutirá, en el presente trabajo, la cuestión del término con que se denomina la lengua. Empleamos la voz "castellano" en el sentido que se le daba en Chile en tiempos de Lenz y que sigue conservando en el lenguaje político-educacional y cotidiano chileno.
} 
El presente estudio se centrará en las ideas de Lenz con respecto, especialmente, a la lengua española en Chile y, más particularmente, a la enseñanza de su variedad "literaria" en la república. Dada la amplitud de dimensiones que abrazó el pensamiento lingüístico de Lenz, es importante precisar que no se intentará, en este estudio, conectar sistemáticamente las ideas del lingüista alemán con el contexto teórico de la época en Hispanoamérica -por ejemplo, con los planteamientos de Cuervo-, ni, menos aun, se buscarán los antecedentes o la matriz científica del pensamiento de Lenz en la tradición filológica y lingüística europea. La investigación pretende contribuir a una mejor comprensión no solo del pensamiento lingüístico aplicado a la enseñanza en esa época sino, también, indirectamente, de los procesos de planificación lingüística implementados en el período, los que siguieron teniendo incidencia mucho después de la muerte de Lenz (v. Flores Gutiérrez 1950).

De acuerdo con la definición de Cooper (1997), en un sentido amplio, la planificación lingüística incluye los diversos "esfuerzos deliberados por influir en el comportamiento de otras personas respecto de la adquisición, la estructura o la asignación funcional de sus códigos lingüísticos" (p. 60). Así concebida, el alcance de la planificación lingüística cubre desde las grandes políticas nacionales diseñadas y ejecutadas por el Estado, hasta la conducta cotidiana de maestros y comunicadores sociales en la escuela o en los medios de comunicación de masas. En todas estas dimensiones, el pensamiento de Lenz estuvo presente, polemizó, muchas veces en forma irónica, y contribuyó, en Chile, a un cambio paralelo, aunque tal vez no idéntico, al realizado más al norte por Cuervo: el paso "de las 'ideas lingüísticas' de hablantes a la exposición rigurosa de hechos de lenguaje ${ }^{4}$ " (Guitarte 1991: 181).

4 Podría objetarse que este cambio ya había tenido lugar con Bello. A diferencia de éste, Lenz no restringió sus estudios a una concepción restringida de lenguaje, sino que, de acuerdo con el pensamiento lingüístico moderno y contemporáneo, comprendió que también eran dignos de estudio el español hablado por los sectores populares y las lenguas indígenas americanas. 


\section{EL CONTEXTO}

El período en que Lenz desarrolla su actividad académica se encuentra a caballo entre los siglos XIX y XX y parece constituir un momento de bisagra en la historia del español en Chile. Comienza con el arribo de Lenz, desde Alemania, en 1890, poco antes de la Revolución del 91, fecha que puede considerarse históricamente como el inicio, o la prefiguración, del siglo XX chileno (v. Subercaseaux 1997, Correa et al. 2001), y se extiende hasta 1938, año del deceso de Lenz, coincidente con el fin de lo que Cartagena (2002) denomina "período de estandarización de la lengua española en Chile"5.

Constituye esta una época de profundos cambios en diversos ámbitos de la vida social chilena. Las transformaciones que ocurren en el fin de siglo se ven potenciadas por la bonanza económica existente. Chile ha triunfado en la Guerra del Pacífico y los tributos impuestos a la exportación del salitre incrementan grandemente las arcas fiscales, lo que permite una fuerte inversión del Estado con fines modernizadores. Esto lleva, a partir del gobierno de Balmaceda, a una importante expansión de los servicios públicos que, a pesar de los cambios dramáticos del 91, continuará durante las décadas siguientes ${ }^{6}$. En efecto, si en 1880 la administración pública contaba con 3.000 funcionarios, éstos aumentarían hasta 13.000 en 1900 y alcanzarían los 27.000 en 1919 (Correa et al. 2001: 24).

El crecimiento fue especialmente notable en la enseñanza pública. Así, por ejemplo, mientras hacia 1880 había 620 escuelas públicas de un total de 1.025 establecimientos de este nivel, para 1901 se había llegado a las 1.700 escuelas públicas sobre un total de 2.159 (Campos Harriet 1960: 30-31). Si miramos los ocupados en la educación pública, de menos de 500 en 1880 se pasa a 12.650 en 1930 (Correa et al. 2001: 24). El período balmacedista (1886-1891) fue especialmente fructífero en su política expansiva, con un aumento superior al $30 \%$ en la cantidad de escuelas primarias y del $50 \%$ en el número de profesores primarios (Ramírez Necochea 1964: 53-54). Además de ello, durante este mismo período se crearon escuelas normales en provincia (Chillán y La Serena); se envió a maestros a especializarse a Europa; se subieron los sueldos de los profesores; se fundaron diez liceos

\footnotetext{
5 Con arreglo a la periodización sugerida provisionalmente por Cartagena (2002), el lapso en estudio corresponde, en gran medida, a la segunda parte de este período, iniciado en 1843

6 Para una caracterización de los cambios económicos y culturales del período balmacedista, véanse Ramírez Necochea (1964) y Subercaseaux (1997).
} 
para la enseñanza secundaria, entre ellos el primero de mujeres ${ }^{7}$; y se modificaron los planes de estudio y programas de la educación secundaria, estableciéndose el denominado plan concéntrico de enseñanza ${ }^{8}$. Con todo, el analfabetismo siguió predominando en la población hasta bien entrado el siglo XX. De acuerdo con Amanda Labarca, en 1895 la tasa de analfabetismo ascendía a $68,2 \%$ y en 1907, más de diez años después, había bajado solo 8 puntos, a un $60 \%$. Todavía en 1920 , la mitad de la población era analfabeta (cit. en Cartagena 2002: 61).

En el marco de estas realizaciones, se establece en 1889 el Instituto Pedagógico de la Universidad de Chile. Como afirma Ramírez Necochea, "[c]on él, se perseguían objetivos bien definidos: el mejoramiento y progreso de la educación secundaria, con la formación de profesores idóneos y especializados" (1964: 55). Para la dictación de cursos en el nuevo instituto, el gobierno decidió contratar a profesores alemanes, uno de estos, Lenz.

\section{QUÉ LENGUA ENSEÑAR: EL PROBLEMA DE LA LENGUA LITERARIA}

Si bien Lenz llegó a Chile para ejercer de profesor de francés, inglés e italiano ${ }^{9}$, a partir de 1895 , y hasta su jubilación en 1925, dictó la cátedra de lingüística española, que tenía como eje la enseñanza de la gramática sincrónica del español, y, tras la muerte de Federico Hanssen en 1919, se hizo cargo del curso de gramática histórica del español. Desde su llegada, Lenz emprendió una serie de actividades de investigación, docencia, planificación y publicidad - preferentemente, esta última en la revista Anales de la Universidad de Chile-, imprimiendo en ellas el pensamiento lingüístico que traía de su formación alemana.

\footnotetext{
7 Para sopesar adecuadamente el aumento, debe considerarse que, con los diez nuevos establecimientos, el país pasaba a contar con 32 liceos secundarios. En otras palabras, en este corto lapso la cantidad aumentó casi en un $50 \%$.

8 Estas transformaciones no estuvieron libres de conflictos. Durante el XIX, las disputas entre los liberales, que propiciaban el crecimiento de la educación pública, y los conservadores, normalmente refractarios en estas materias, fueron constantes. En general, la disputa estuvo también atravesada por la cuestión doctrinaria del papel de la Iglesia en la enseñanza (véanse Campos Harriet 1960; Jaksic y Serrano 1990).

9 Finalmente, no se instaló la cátedra de italiano.
} 
Lenz abordó también la cuestión de la enseñanza del castellano como idioma patrio, focalizándose en el problema de la enseñanza de la "lengua literaria". En este marco, se abocó a tres cuestiones que, evidentemente, consideraba centrales: la determinación de la "lengua literaria" que debía enseñarse; el método con que esto se realizaría -el así llamado "directo"-; y, por último, estrechamente ligado a lo anterior, el papel que desempeñaría la gramática en esta tarea. Respondiendo al espíritu unitario que Ferreccio (s.f.) reconoce en el autor alemán, las ideas de Lenz con respecto a este tema se integraron en una concepción más amplia, que buscaba ser consistente con su noción de "lenguaje vulgar", y se proyectaron no solo en la labor docente sino también en la investigación científica, los informes tras visitas a liceos, el diseño de programas oficiales de enseñanza del "idioma patrio" y la divulgación y publicidad dirigida a la audiencia "culta" chilena.

La enseñanza del español "literario" constituía, para Lenz, la tarea fundamental de la educación pública de la época. "La enseñanza del castellano es el ramo más importante de toda la instrucción secundaria", escribirá en su Memoria sobre las tendencias de la enseñanza del idioma patrio en Chile $(1899$, p. 3), texto de trinchera dirigido a la audiencia nacional. Precisamente su función, como maestro del Instituto Pedagógico, era preparar para esta labor a los profesores que impartirían clases en la enseñanza secundaria, nivel reservado, en ese entonces, a una minoría en el país. A este papel se agregaron cierta función de fiscalización y superintendencia del quehacer de los liceos secundarios ${ }^{10} \mathrm{y}$ un rol de más proyección en la reforma de la enseñanza del "castellano".

A juicio de Lenz (1899), la enseñanza del "idioma patrio" cumplía cinco funciones: "adiestrar al alumno en la lójica práctica"; "darle nociones literarias"; "formar su carácter"; favorecer la adquisición de "todos los conocimientos especiales" que entrega el liceo, especialmente en las humanidades; y servir de "ayuda a la enseñanza del idioma [...] estranjero" (p. 4). La idea de Lenz era que, durante sus estudios escolares y de liceo, los estudiantes debían pasar del "habla vulgar i dialéctica [=dialectal]", a la "lengua literaria castellana". Como es evidente para cualquiera que se haya acercado mínimamente a su obra, esto no significaba en Lenz un desprecio por esta "habla vulgar", que le parecía la expresión más genuina de la

10 Específicamente, fue "visitador de liceos". Su función consistió en "vigilar la aplicación adecuada de los nuevos métodos humanísticos implantados en los establecimientos secundarios de toda la República" (Parada 1954: 3-4). 
lengua" ${ }^{11}$ La variedad "literaria", aunque "artificial", tenía sentido como una herramienta que facilitaba el desempeño en la dirección de los asuntos públicos y económicos del país, y el acceso a la alta cultura, tanto patria como de "los pueblos de habla castellana en general" (p. 3).

El primer problema para Lenz radicaba en precisar qué se entendería por "lengua literaria" y cómo ésta resultaría compatible con su noción de "lenguaje vulgar" en un sistema conceptual coherente que debía, a la vez, proponer una lengua estándar y reconocer la existencia de variedades dialectales. En su Memoria de 1899, Lenz reconoce que "todo lenguaje de un hombre adulto es correcto dentro de su esfera" (p. 6). En este sentido, la oposición entre "lenguaje vulgar" y "lengua literaria" no se podía fundar en la absolutización de la dicotomía correcto/incorrecto como era usual en Chile por esa época. La posición de Lenz a este respecto es clara y se mantiene a lo largo de toda su obra: la lengua constituye una trama compleja de diversas variedades, todas ellas legítimas en algún ámbito.

Desde el punto de vista genético, la "lengua literaria" obedece, como expone en el prólogo del Diccionario Etimolójico de las voces chilenas derivadas de las lenguas indígenas americanas (1910) ${ }^{12}$, a razones "históricas de política": la instauración de un gobierno central que rige sobre un territorio extenso, la difusión de la burocracia administrativa, el empleo de la escritura y el surgimiento de una literatura modélica. Por las funciones que está llamada a desempeñar, su uso se encuentra, preferentemente, en la comunicación pública y entre las personas de cierta educación:

Creo que en un estado civilizado moderno usan normalmente la lengua literaria todos los que han estado en un establecimiento de educación secundaria; i la expresión más lejítima del lenguaje escrito de cada nación debe buscarse en lo que mas se lee: en el diario, en la prensa periódica, que abarca no solo la literatura corriente sino además toda la vida moderna (1910 [s.f.]: 12).

11 Para muestra, una breve cita: "El mismo lenguaje dialéctico del bajo pueblo espresa mucho mas fielmente la vida intelectual de la actualidad i de los siglos pasados de lo que lo hace el lenguaje artificial literario" (Lenz 1894a: 355).

12 Recordemos que la primera parte del Diccionario se publicó, para circulación privada y reparto oficial, en septiembre de 1905, y que su impresión había tardado más de un año. En este sentido, si consideramos que el proyecto debe haberse formulado al menos un año antes de su ejecución, las ideas contenidas en el "Prólogo" deberían haber estado claras ya en 1903. Refuerza esta hipótesis, el carácter extremadamente sistemático del Diccionario, que deja ver su sujeción a un plan que el autor ha de haber madurado por bastante tiempo. 
Si bien para Lenz los conceptos de "lenguaje vulgar" y "lengua literaria" debían integrarse en una totalidad coherente, la relación entre ambos no era simple. Ferreccio (s.f.) llegará a decir, con respecto al "Prólogo" del Diccionario, que los intentos de Lenz por delimitar el "castellano" como lengua general de cultura, distinguiendo lo común de lo regional y dialectal, "desembocan en un monumental embrollo" (p. 14). Vale la pena detenerse brevemente en las distinciones, de claro valor programático, que Lenz propone en este prólogo, pues, por su propósito de fundamentar el estudio lexicográfico científicamente, en él se presenta el problema de manera más detallada que en los textos destinados a la enseñanza del "idioma patrio" en Chile.

En el "Prólogo", el lingüista alemán parte por establecer, centrándose en el dominio del léxico, una distinción básica entre la "lengua jeneral" o común y los dialectos. La lengua general estará constituida, en su centro, por el "lenguaje nacional", que se define como "el lenguaje cotidiano de la vida pública i social escrito i hablado por toda la jente culta" (p. 12). Hacia arriba, se ubicará el "lenguaje literario" (en otra parte habla de "estilo literario"), correspondiente a la lengua escrita y, por ende, "las bellas letras, las ciencias i artes, la industria i el alto comercio" (p. 12). Hacia abajo, estará el "lenguaje familiar", de carácter oral, y concerniente a la vida doméstica. Lo propiamente "dialectal" corresponderá, al menos en el léxico, a los términos que se emplean exclusivamente por la gente sin educación escolar. Habrá, finalmente, un léxico "provincial", constituido por términos que, si bien utilizan las personas educadas, coexisten con otros que poseen el mismo significado y un uso más extendido.

La categorización propuesta presenta dos aspectos problemáticos. En primer lugar, y esto es ciertamente un detalle, la extensión asignada al término "lenguaje literario" resulta aquí menor que la del concepto "lengua literaria", que Lenz ha usado antes y que seguirá empleando en otros textos. Como quedará claro al revisar otros escritos del autor, la "lengua literaria" corresponde, en términos generales, a lo que hoy llamaríamos el estándar, y abarca no solo lo escrito sino también lo oral. De este modo, al menos el núcleo -el "lenguaje nacional"- o parte de él, y la capa superior de la lengua común -el "lenguaje [o estilo] literario")- constituyen, a nuestro entender, lo que Lenz denomina, en sentido amplio, "lengua literaria", es decir, la variedad que debe enseñarse en el sistema educacional chileno. Por supuesto, tanto en la construcción social de la "lengua literaria" como en la instrucción secundaria, el modelo del estándar vendrá dado, preferentemente, por los textos del estilo literario; no obstante, habrá también lugar para la ortología.

El segundo problema radica en el empleo del concepto de "nación", al definir el núcleo de la lengua común. La posición de Lenz sobre la relación entre lenguaje, nación, etnia y Estado es compleja y la he tratado en otra 
parte (Soto 2015). En el texto en comento plantea que la lengua común parte estableciéndose dentro de los límites del Estado-nación, como "idioma patrio" de un grupo étnico: "El chileno no habla mas que un solo idioma, el castellano-chileno" (1910 [s.f.]: 42). Esto implica que aquí, para Lenz, habrá una "lengua literaria", es decir, estándar, chilena que podrá ser, en algunos aspectos, distinta de, por ejemplo, la peninsular. Las diferencias no serán vistas, en este caso, con alarma sino con la misma tolerancia con que se acepta en Inglaterra que un libro editado en los Estados Unidos se distancie en algo de la norma británica. La unidad idiomática del español no descansará sobre una "uniformidad absoluta del idioma" que, de hecho, "no existe en ninguna parte del mundo" (Lenz 1899: 8). A modo de ejemplo, señala, todavía en 1899, que si se observa el inglés se descubrirá que existen algunas diferencias de dicción y construcción entre el de Nueva York, el de San Francisco, el de Sydney y el de Calcuta. Esta situación es también normal en otras lenguas europeas como el alemán, el italiano y el francés. No obstante, estas diferencias no debieran interpretarse en el sentido de una fragmentación del patrimonio común, como había temido Bello y deseado Sarmiento: "[...] i sin embargo los yankees nunca han manifestado temor de que pudieran perder la lengua inglesa ni se ha reprochado a los vieneses que no hablen alemán" (p. 8). De hecho, en el "Prólogo" explícitamente reconoce la existencia de lo "pan-americano" y lo "pan-castellano", solo que, prudentemente, considera que la determinación de estas entidades deriva de investigaciones previas de alcance nacional.

A nuestro juicio, esta concepción flexible le permitía a Lenz conciliar diversos supuestos. En primer término, era compatible con la idea de que, finalmente, el español, por razones históricas, no presentaba mucha variedad entre los distintos pueblos que lo hablaban; menos aun, como había dicho Bello, entre la gente educada -cabe precisar que Lenz abandona luego su hipótesis radical sobre la singularidad del "lenguaje vulgar" chileno-. En segundo lugar, salvaba cierto compromiso nacionalista puesto que las fronteras del Estado-nación, en el caso chileno, constituían el primer ámbito de determinación de la "lengua nacional". En este sentido, se garantizaba la apropiación de la lengua por parte de la nación organizada, $\mathrm{y}$, consecuentemente, se legitimaban en doctrina las políticas lingüísticas llevadas a cabo por el Estado nacional sin caer en la pretensión de un Abeille, de cuya obra, decía, hay que precaverse ${ }^{13}$. Finalmente, la concepción

13 El francés L. Abeille publica, en 1900, El idioma nacional de los argentinos. Según Lenz, el libro "está lleno de ciencia postiza". A pesar de que algunos pasajes de sus obras, particularmente las publicadas en Chile con fines de publicidad, puedan sugerir lo contrario, 
consideraba los aspectos políticos y sociales que están detrás de la definición de la "lengua literaria" como un objeto cultural, en cierto modo, "artificial", como repite Lenz en diversos escritos. Dada la naturaleza constructiva de este objeto, y su carácter eminentemente funcional, Lenz tenía conciencia de que se trataba de una entidad normativa, que, en gran medida, respondía a necesidades prácticas.

En una obra tardía, dirigida a la comunidad científica hispanohablante y publicada en España, La oración y sus partes, Lenz continuará sosteniendo esta concepción tolerante con respecto a las diferencias y prudente en relación con la determinación de lo "pan-castellano". No obstante, ya no hablará de lenguas literarias nacionales:

Una lengua literaria, hablada en tan dilatados territorios como la castellana, no puede ser completamente uniforme en todas partes. Lo que necesitamos, ante todo, es saber cuál es el uso efectivo del lenguaje familiar, tanto de la gente culta como del pueblo de poca o ninguna cultura literaria, no sólo con respecto al vocabulario (que es lo que más varía), sino también con respecto a la gramática. Sólo cuando conozcamos bien el lenguaje natural y vivo de todos los países castellanos, podremos escribir la gramática preceptiva del estilo literario sin miedo en recurrir a recomendaciones prácticamente utópicas (1920 [1935]: 32).

No queremos dar a entender acá que Lenz hubiera diseñado, hacia fines del siglo XIX, un sistema donde "lenguaje vulgar" y "lengua literaria" se integraran con absoluta claridad. Ambos conceptos no solo se ubicaban en los dos extremos de la lengua, sino que, además, pertenecían a ámbitos distintos. Mientras el "lenguaje vulgar" mostraba el uso lingüístico en su estado puro, como mero resultado de leyes científicas, la "lengua literaria" era el producto de un proceso de estandarización (aunque el término no figura en las obras consultadas), construido políticamente en el marco del Estado. De un lado, el "lenguaje vulgar" era la expresión más genuina del espíritu del pueblo; del otro, la "lengua literaria" era el resultado del desarrollo social y cultural de un Estado que descansaba sobre ese pueblo. Esta relación entre pueblo y Estado llevaba a que no pudiera simplemente renunciarse al estudio de las formas dialectales del lenguaje ni al diseño e implementación de políticas públicas tendientes a fijar un estándar nacional. Con todo, este principio no opera de manera absoluta en Lenz, pues reconoce una unidad lingüística

Lenz es claro en no identificar lengua con nación ni raza. Su posición en este ámbito es próxima a la de Renan (Alfón 2011, Soto 2015). 
supranacional que, aunque no sea monolítica, abarca los pueblos que en América y Europa hablan y escriben en "castellano". Esta unidad obedece a la historia de la expansión del español y es sobre todo patente en la lengua escrita, aunque también se manifiesta en la oralidad.

Las ideas de Lenz con respecto a la "lengua literaria" se contraponían a los planteamientos que había sostenido anteriormente Andrés Bello, los que, a fines del siglo XIX, parecían formar parte del sentido común de los sectores ilustrados. Como se sabe, el intelectual venezolano trató el problema del estándar en las "Nociones preliminares" de su Gramática de 1847, donde se refirió al "buen uso", en el sentido del habla de la "gente educada"14. Bello justificaba su definición en un criterio comunicativo que, en principio, parecería neutro con respecto al tema de la identidad idiomática ${ }^{15}$. En efecto, el privilegio acordado al buen uso obedecía, al menos en forma expresa, a una decisión puramente racional: "es el más uniforme en las varias provincias y pueblos que hablan una misma lengua, y por lo tanto el que hace que más fácil y generalmente se entienda lo que se dice" (1847 [1928]: § 2(a)).

Con todo, la aplicación en Chile de las ideas lingüísticas de Bello parece haber conducido a actitudes y prácticas puristas. Ciertamente, no corresponde juzgar a Bello por sus epígonos; sin embargo, el origen de esta actitud puede observarse en la ya mencionada preocupación del intelectual venezolano con respecto a una eventual fragmentación del castellano, $\mathrm{y}$, consecuentemente, en su afán por conservar "la lengua de nuestros padres en su posible pureza" (p. vii).

Para Bello, la lengua estándar se vinculaba fuertemente a la tradición peninsular. Gómez Asencio (2009) ha hecho un recuento y un cálculo -"aproximativo", dice- de los autores que figuran en los ejemplos de la Gramática de Bello, esto es, aquellos que ésta considera modelos del buen uso del español. Salvo unas pocas excepciones, Bello emplea autores españoles, predominantemente no meridionales. Un $60 \%$ de éstos son anteriores a 1750 ; un $20 \%$ se ubican en el período de 1750 a 1800 y tan solo el $20 \%$ restante corresponde a autores coetáneos a Bello. Como es de esperar, el más citado es Cervantes.

14 Lenz critica el recurso a la "gente educada". Para él, el tema era la ilustración de tipo intelectual y no la educación, que vinculaba con lo moral (v. Lenz 1912 [1955]).

15 Por cierto, el criterio comunicativo no era absoluto en la concepción que Bello tenía del español como lengua común a España y las naciones hispanoamericanas. Reconocía en él dos funciones: servir de "vínculo de fraternidad", en este caso supranacional, y de "medio providencial de comunicación” (1847 [1928]: p. vii). 
En su Memoria de 1899, en cambio, Lenz precisa el concepto de "lengua literaria", descartando de plano las interpretaciones puristas. En efecto, parte señalando que nadie consideraría como tal la empleada por los grandes autores del Siglo de Oro, pues ésta "no serviría para espresar nuestros pensamientos modernos" (p. 7). Tampoco acepta la lengua empleada por los buenos autores hispanos. Al respecto, escribe: "[e]so sería aceptable si fuéramos españoles, pero se trata del pueblo chileno i de los americanos de descendencia española en jeneral" (p. 7). Contra la idea bellista de los hispanoamericanos como defensores de la tradición peninsular, Lenz enfatiza como criterio el uso real, y la norma, hoy diríamos estadística, como parámetro de corrección:

El término medio de las palabras i construcciones usadas en una lengua representan el "uso correcto" exista o no un libro que codifique en fórmulas abstractas cuáles son las construcciones usadas i otro libro que contenga todas aquellas palabras; es decir, existan o no existan escritos la gramática i el diccionario (1899: 6).

La idea seguiría presente por muchos años en Lenz. Así, de acuerdo con los apuntes de su curso de lingüística general, publicados por María Luisa Jenschke, su alumna, para Lenz la lengua literaria "es más o menos el término medio de lo que prefiere cierta mayoría de la jente" (1923: 66-67).

En conclusión, si Bello pensaba en una lengua común, cuya identidad residía en la tradición peninsular, y respecto de la cual todos los hispanohablantes "educados" de mediados del XIX tenían los mismos derechos -en la medida en que respetaran su genio-, Lenz pensaba más bien, como hemos visto, en una "lengua literaria" general, no monolítica, en la que coexistían diversas "lenguas literarias" nacionales, bastante semejantes entre sí, correspondientes a los distintos estados en que se hablaba español y determinadas, en la práctica, por el uso de sus contemporáneos. En otras palabras, para Lenz parecía no haber contradicción entre la unidad "pancastellana" y la unidad nacional: la tolerancia ante diferencias que, en todo caso, no eran mayores permitía abrazar ambos ideales.

Como puede apreciarse, Lenz relaciona sus planteamientos con la idea de nación, aunque en este caso - para ser rigurosos- no es siempre evidente que esté pensando esta noción a partir del complejo raza-etnia-lengua. Lo que sí es claro, es que con sus ideas propugna una suerte de declaración de independencia con respecto a la implementación de políticas lingüísticas:

Ojalá, que al fin los hispano-americanos tuvieran suficiente respeto con su propia nacionalidad para desistir de esa devoción a una autoridad española que es todo menos competente en materia lingüística. Ojalá que 
los chilenos, admirados en América latina por su ardoroso patriotismo, fueran el primer pueblo que echara a un lado las añejas i rancias ideas que todavía dominan a la mayor parte de los literatos i de los profesores de castellano (1899: 8-9).

Esta independencia, que sin embargo reconoce una unidad idiomática superior, se ve facilitada por el hecho de que Lenz no considera a la Real Academia Española, hacia 1900, una entidad científica respetable, y no admite, por tanto, su papel rector. El juicio condenatorio alcanza a casi toda la lingüística y la filología española de la época, pero es especialmente duro con el diccionario académico, que para los chilenos de entonces encarnaba la norma de corrección:

Diccionario de Real Academia felizmente no lo hai ni en inglés ni en alemán, i sin embargo, no se ha perdido la unidad lingüística de esos dos grandes grupos de pueblos jermánicos esparcidos sobre el orbe entero en número de más de ciento cincuenta millones (1899: 8).

Las críticas a la labor lexicográfica de la Real Academia Española son repetidas. Así, en el "Prólogo" de su Diccionario escribe, comparando implícitamente su obra con la académica:

No aspiro a que la Real Academia Española tome nota del fruto de mis desvelos para decidirse a aceptar en el Léxico oficial alguna voz que hasta hoi no figura en él.

Mientras esa corporación no comience su diccionario con un prólogo en que esponga con claridad según qué principios admite i escluye voces, no sabria realmente qué provecho podria sacar de mi diccionario (1910 [s.f.]: 40).

En una nota al pie de esa misma página, la opinión es más tajante aun:

Así como es el Diccionario de la Academia no es ni un diccionario literario (que deberia escluir todo lo que no se pueda usar por escrito) ni un diccionario completo de toda la lengua; es un libro sin principios científicos claros.

Con todo, más adelante morigerará su crítica en relación, al menos, con la doctrina gramatical de la Real Academia, y llegará a propugnar con éxito la sustitución, en la enseñanza, de la terminología de Bello por la de la gramática académica, que le parecerá, entonces, científicamente más adecuada y de mayor aceptación en el mundo "pan-castellano". 
Quizás donde con mayor nitidez se observe la tensión entre el carácter a la vez nacional y "pan-castellano" de la "lengua literaria" sea en los dominios de la pronunciación y de la ortografía. En 1894, Lenz publica sus "Apuntaciones para un testo de ortolojía i ortografía de la lengua castellana", escritas a petición de Federico Puga Borne y publicadas en el marco de la polémica ortográfica que, como se sabe, atravesaría la historia lingüística chilena hasta entrado el siglo XX. Estas "apuntaciones" dejan en claro que para Lenz la "lengua literaria", aunque fundada en lo escribible, no se limitaba tan solo al español escrito. Un examen somero de las recomendaciones que da Lenz sobre la pronunciación nos permiten apreciar su idea de "corrección" idiomática con respecto a la "lengua literaria".

La primera censura de Lenz afecta a los cambios de acentuación y de timbre en los grupos aí, aú, eí y oí. Para Lenz, su sustitución por diptongos del tipo óido no es correcta, "ni mucho ménos la pronunciación éi por ai (méi, péi, éi por maíz, país, ahi), que es tan comun en el bajo pueblo de Chile" (1894b: $\S 21)$. Con respecto a las consonantes, le parece que no tiene sentido reimplantar la distinción entre $/ \theta /$ y $/ \mathrm{s} /(<$ caza $>$ vs. $<$ casa $>)$; critica la supresión de /d/ en participios y en posición posnuclear; condena la pérdida de la distinción entre la lateral palatal y la fricativa palatal (pollo vs. poyo); rechaza la confusión entre $/ \mathrm{r} / \mathrm{y} / \mathrm{l} /$ en posición posnuclear; $\mathrm{y}$ censura la elisión de /s/ ante consonante y en final de palabra, y la asimilación que provoca la /s/ aspirada de la consonante que la sigue (refalar por resbalar).

En general, los mismos juicios ya habían sido emitidos anteriormente por Bello en sus Advertencias (1833-1834 [1940]) y sus Principios de ortolojía $(1837[1875])^{16}$. Más novedosas resultan la crítica de la asibilación del grupo /tr/, y, sobre todo, la censura de una realización de alcance completamente general en el español de Chile: la palatalización de las velares ante vocales anteriores. Escribe Lenz al respecto: "sobretodo téngase cuidado de no pronunciar yerra, yinda en vez de guerra, guinda" (1894b: § 26); "no se pronuncia quie, quieda sino que queda" (§ 28); "ni se debe decir jiente en lugar de jente" (\$ 31).

La selección de las críticas muestra que Lenz no consideraba solo el uso nacional para la determinación de la pronunciación estándar. Si bien no aconseja introducir la distinción $/ \theta / \mathrm{y} / \mathrm{s} /$, que no se da en América, y critica la confusión entre / $\theta /$ y /1/, que ya Bello califica de vulgarismo (1833-1834 [1940] § 30), censura la palatalización de velares ante vocales anteriores,

16 Con respecto a la distinción / $\theta /-$ /s/, Bello la recomienda, en sus "Advertencias", como propia de "una pronunciación más esmerada" (§ 30). En los "Principios", en cambio, plantea que intentar que esta distinción se emplee en América es "cosa ya desesperada" (p. 18). 
un empleo extendido incluso entre los sectores de mayor educación y sobre el que probablemente no existía conciencia por parte de los hablantes, $y$, en consecuencia, no estaba estigmatizado ${ }^{17}$. Por supuesto, difícilmente Lenz habría llegado a plantear, como Menéndez Pidal, que se impusiera la enseñanza de la pronunciación de Castilla a los extranjeros (Del Valle 1999); no obstante, al parecer sus opiniones no se fundaban solamente en el término medio de los chilenos educados: entre lo nacional y lo "pan-castellano" parecía ubicarse, por una parte, lo "pan-americano", y, por otra, criterios históricos como parece ocurrir en la defensa de la distinción entre la lateral palatal y la fricativa palata ${ }^{18}$.

En cuanto a la ortografía, las "Apuntaciones" de 1894, de acuerdo con el propio Lenz, se ajustan, en términos generales, a la de Bello, aunque en algunos casos se prefieren usos americanos corrientes (estraño y no extraño) e innovaciones de la Real Academia (virrei y no virei). Un aspecto interesante lo constituye la mantención de la distinción entre $<\mathrm{z}>,<\mathrm{c}>$, de una parte, $\mathrm{y}$ $<\mathrm{s}>$, de otra, que, como se sabe, en el español de América no corresponden a ninguna diferencia en la pronunciación. Aunque Lenz, en este texto por encargo, no innova, no puede evitar una decidora nota de pie de página:

Sería de desear que los americanos escribieran siempre $s$ cuando la pronuncian; la ortografía no se haría por esto menos intelijible, porque de verdad nadie creerá que ellos no se entiendan cuando hablan, aunque no hacen diferencia en la pronunciación (1894b: 120).

Por la misma razón, también plantea que sería razonable terminar con la distinción entre $<\mathrm{b}>\mathrm{y}<\mathrm{v}>$. Con respecto a la $<\mathrm{h}>$, sugiere que, tal y como han hecho los italianos, se la elimine.

Dos razones pueden explicar el que las "Apuntaciones" no fueran más allá de las sugerencias. La primera, y probablemente la más importante, es que se trataba de un texto por encargo, cuya publicación en 1894 respondía al interés de la Facultad de Filosofía y Humanidades por uniformar la ortografía, y en el que se atacaban, a la vez, dos flancos: por un lado, se trataba de "adelantar un paso mas hacia el ideal de toda ortografía, el fonetismo" (p. 108), y, por el otro, se entregaba a los profesores "todo el material que, según mi opinión [la de Lenz, claro], debería enseñarse en los liceos i hasta

17 Si bien no conocemos investigaciones en torno a las actitudes lingüísticas adscritas a este fenómeno, es probable que hoy no haya conciencia del mismo por parte de los hablantes chilenos.

18 Este caso es interesante, además, porque las zonas no distinguidoras eran precisamente Santiago y sus alrededores. 
cierto grado tambien en las escuelas primarias" ${ }^{19}$. La segunda razón es que Lenz estaba consciente de que la reforma ortográfica constituía, finalmente, una medida tomada desde la autoridad que entraba en conflicto con el uso. Si bien esto no afectaba el proyecto, científicamente fundado, de establecer una ortografía fonética, sí hacía necesaria la implementación gradual de esta reforma:

La única norma verdadera para la ortografía es la pronunciación; pero no se puede negar toda influencia al uso, que siempre es conservador, porque cada innovación es desagradable durante algun tiempo. El uso exije que en todas las reformas ortográficas se proceda sin nada de exajeración; por esto cuando ese uso conserva letras etimolójicas superfluas que dificultan el aprendizaje de la ortografia, hai que desterrarlas poco a росо $(\S 9)$.

La concesión al uso era, evidentemente, política. Como hemos dicho, Lenz confiaba en que la reforma se impondría, y probablemente consideraba que estaban a favor de ésta tanto el carácter científico que le atribuía, como su idea de que el estado chileno tenía el suficiente poder como para imponer este cambio. Finalmente, no era sino una cuestión de tiempo. El progreso estaba a su favor:

La ortografía castellana va a cambiarse i reformarse en el porvenir, como todas las ortografías de las lenguas cultas, hasta que alcance el ideal, la ortografía fonética, que para cada sonido distintivo del lenguaje cuidado, tiene un solo signo gráfico. Tales reformas, como por ejemplo la supresión completa de las letras $x$ i $h$, deben prepararse durante años (p. 109).

\section{CÓMO ENSEÑAR CASTELLANO EN EL LICEO: GRAMÁTICA VS. PRÁCTICA DEL IDIOMA}

Como hemos dicho, junto con determinar qué "lengua literaria" se enseñaría, Lenz se preocupó también de cómo se realizaría la instrucción en los liceos y qué papel desempeñaría la gramática en este proceso. En estos puntos, nuevamente entraría en conflicto con las ideas heredadas de Bello. Dos fueron

19 Por supuesto, este material no era solo ortográfico. De hecho, Lenz indica que son mayores las innovaciones en materias ortológicas. 
sus discrepancias con el erudito venezolano. En primer lugar, a diferencia de Bello, Lenz consideraba que la gramática no desempeñaba una función muy importante en la enseñanza de la "lengua literaria". Contra el privilegio acordado a la gramática, Lenz planteaba la necesidad de un estudio práctico de la "lengua literaria", no abstracto, consistente en la exposición a modelos, el ejercicio imitativo y la "práctica constante i dirijida por la mano del maestro que conoce la teoría i el desarrollo histórico" (1899: 5). En lugar del libro de gramática debía estar el de lectura.

La postura metodológica de Lenz descansaba en el supuesto de que los principios de aprendizaje semejantes eran válidos, en términos generales, para la adquisición de cualquier tipo de lengua (materna, extranjera, "literaria"). Lenz opera aquí con una cadena de razonamientos analógicos. Si los niños adquieren su lengua materna mediante el solo recurso de la práctica, sin necesidad de que se les enseñen explícitamente reglas gramaticales ni teorías lingüísticas, de modo similar los jóvenes pueden aprender una lengua extranjera sin centrar esta tarea en el estudio teórico de su gramática. Asumiendo esta hipótesis, y considerando que la relación entre el "lenguaje vulgar" y la "lengua literaria" es similar a la que se da entre la lengua materna y una extranjera, se desprende la proyección del "método directo" a la clase de idioma patrio. Lenz mantuvo esta posición durante toda su vida académica:

Sería un absurdo que no se reconociera definitivamente que la lengua patria se debe aprender, i se puede aprender únicamente por la práctica, lo mismo que los idiomas estranjeros. Los que creen que el estudio teórico de la gramática tiene importancia mayor para conseguir el dominio sobre la lengua literaria según mi opinión, están simplemente equivocados (1920: 350).

No obstante lo anterior, el "método directo", centrado en los buenos modelos y el ejercicio práctico, no operaba, en Lenz, como principio absoluto. Y esto, a pesar de la reforma de la enseñanza secundaria de 1893, que, siguiendo innovaciones de la educación alemana, había decretado que "el idioma patrio se debe aprender por imitación de buenos ejemplos i no por estudios teóricos" (Lenz 1924: 1243) ${ }^{20}$. Lenz consideraba que el estudio de la gramática no era del todo superfluo para la enseñanza de lenguas $-\mathrm{y}$ en este punto, tendrá el

20 En rigor, la reforma de 1893 había obedecido a un programa propuesto por Hanssen que, conforme a los programas vigentes en Alemania, ponía "en primer término la práctica del idioma y reducía la enseñanza teórica gramatical a un mínimo, recomendando repetidas veces que en los años inferiores se debían evitar las definiciones" (Lenz 1912 [1955]: 13). 
lingüista alemán una clara discrepancia con su discípulo Saavedra Molina, quien extremaba el compromiso con el método práctico ${ }^{21}-$ :

el conocimiento consciente de la gramática teórica es completamente superfluo para 'hablar' cualquier idioma; tiene utilidad el estudio de la 'teoría del lenguaje' (o gramática) cuando se trata de 'escribir', i en mayor grado, cuando se trata de aprender en el colejio una lengua estranjera (1920: 351).

Esta aceptación de un papel, ciertamente menor, de la gramática en la enseñanza, derivaba de que, a pesar de las analogías, los diversos procesos de adquisición o aprendizaje no eran, para Lenz, exactamente iguales. Mientras "el lenguaje corriente de la conversación" se adquiría naturalmente, sin estudio teórico, "el manejo de una lengua literaria" era un arte que debía enseñarse en la escuela y el liceo (1899).

La segunda discrepancia con Bello, estrechamente ligada a la anterior, tiene que ver con la definición de gramática. Como se sabe, Bello presenta en su Gramática dos definiciones de este concepto. Por una parte, en el "Prólogo" ésta se considera como la teoría del idioma: "cada lengua tiene su teoría particular, su gramática" (1847 [1928]: 1-2); por otra, al momento de definirla, en las "Nociones preliminares" la caracterización cambia: "La gramática de una lengua es el arte de hablarla correctamente, esto es, conforme al buen uso, que es el de la gente educada" (§ 1). Precisamente esta segunda definición estaba a la base de la idea, aceptada en Chile al momento de llegar Lenz, de que mediante la enseñanza de la gramática podían aprenderse las lenguas. Lenz dedicará varias obras, preferentemente de divulgación, a atacar este punto $(1899,1912$ [1955], 1920, 1924), llegando, incluso, a tratar el tema en el inicio de La oración y sus partes:

Muchas personas, tanto en España como en América, creen aún hoy que las clases de gramática han de enseñar al niño el uso correcto del idioma patrio, o más bien de la lengua literaria nacional, en oposición al lenguaje familiar regional; pues piensan buenamente que la "gramática es el arte de hablar y escribir correctamente". Entre personas que hayan puesto alguna atención a los estudios de filología no habrá necesidad

Hubo también un programa alternativo, de Nercasseau y Morán, que, según Lenz, tuvo escasa aplicación en los liceos fiscales.

${ }_{21}$ La disputa se entrega en un mismo volumen de los Anales de la Universidad de Chile: Lenz (1918), Saavedra (1918). 
de insistir en que tal definición, por venerable que sea su antigüedad, es científicamente inadmisible (1920 [1935]: 23).

Si el manejo correcto de la "lengua literaria" podía considerarse efectivamente un arte, la gramática era una teoría, como ya había afirmado en su Memoria de 1899, que trataba de la enseñanza del "idioma patrio" en Chile. En su texto de batalla Para qué estudiamos gramática, la opinión se da de modo directo:

La gramática no es, ni ha sido jamás, un arte en el sentido de la palabra; la gramática es una ciencia. Hablar una lengua es una costumbre que se adquiere paulatina e inconscientemente, hablar bien eso sí que es arte. ¿Desde cuándo se aprende un arte estudiando una ciencia? (1912 [1955]: 24).

En este punto, Bello había cometido "un profundo error". Y este error de considerar la gramática un arte explicaba su creencia de que había que "incluir la gramática en el plan de estudios secundarios" a fin de "acercar el lenguaje escrito de la gente culta de Chile al tipo general del castellano" ( $p$. 21). La gramática pertenecía al ámbito de las ciencias, y, en consecuencia, debía entenderse como

[1]a espresión abstracta de todos los fenómenos jenerales de la lengua en lo que se refiera a la estructura i variacion de la palabra (fonética y morfología) i a la construcción de las palabras i la frase (sintáxis) (1899: 7).

Por su carácter abstracto, la gramática, según Lenz, no podía enseñarse seriamente en la educación primaria y su estudio efectivo debía posponerse a los últimos años de la secundaria, donde contribuía al ejercicio mental y a la mejor comprensión de la psicología humana:

En la escuela primaria, la enseñanza de la teoría gramatical debe limitarse a un mínimo razonable, atribuyendo toda importancia a los ejercicicos prácticos [...]; también en los años inferiores y medios de la instrucción secundaria conviene evitar las definiciones difíciles, y preparando a la vez un tratamiento más científico para los años superiores. Sólo durante este último período de la enseñanza secundaria puede hacerse un estudio serio, científico, de la gramática del idioma patrio; porque este estudio debe hacerse desde el punto de vista de la gramática general o filosófica [...] (1920 [1935]: 25).

Como ya hemos visto, el Estado chileno aceptó rápidamente la nueva metodología de enseñanza de la "lengua literaria" y el papel que ésta asignaba a la gramática en ella. No obstante, Lenz debió continuar divulgando sus 
ideas con el fin de consolidarlas en una sociedad que, al parecer, había aceptado casi por completo las propuestas de Bello y donde el Estado docente no tenía la hegemonía absoluta de la enseñanza.

\section{QUÉ GRAMÁTICA ENSEÑAR}

Finalmente, junto con precisar el lugar de la gramática en la enseñanza secundaria, Lenz dedicó esfuerzos a la modernización de las doctrinas gramaticales tanto en el campo teórico como en su aplicación en la enseñanza del "idioma patrio". Toda vez que este tema trasciende, en su completitud, los objetivos centrales del presente trabajo, no haremos más que una revisión somera del mismo. Como puede ya predecirse, encontramos acá también diversas constantes del pensamiento del lingüista alemán: su vocación por la investigación y la innovación científicas; su referencia constante a Europa, y en especial a Alemania; su redacción de obras originales y de divulgación; su interés por la aplicación de las nuevas teorías en la enseñanza secundaria; y, por cierto, su crítica a las doctrinas imperantes en Chile, representadas por la Gramática de Bello.

Al parecer, Lenz debió enfrentar, en este ámbito, un contexto más bien reacio al cambio, que consideraba intocables las doctrinas gramaticales del erudito venezolano:

[...] los profesores de castellano en Chile suelen considerar la Gramática de don Andrés Bello como una especie de catecismo cuyos dogmas se deben creer, so pena de ser condenado como hereje. Esto no es cuerdo, no es científico. Bello mismo, que declaró que la única autoridad en materia de lenguaje que reconocía era la lengua misma, no aprobaría tal procedimiento, si viviera (1906: 42).

Por ello, no se limitó a introducir las innovaciones teóricas en sus cursos, sino que desarrolló una extensa actividad de publicista. En sentido estricto, esta tarea de divulgación de los avances científicos ya la había iniciado en 1892 con la publicación de un artículo titulado "La Fonética", en los Anales de la Universidad de Chile. Con seguridad, sus lectores advirtieron inmediatamente que la breve exposición que hacía Lenz de la "ciencia de los sonidos de la voz humana" era muy superior a los viejos Principios de ortolojía y métrica de Bello. Sin embargo, la gramática -en su acepción restringida de morfología y sintaxis- era otra cosa: 
Yo comprendí luego que no bastaba que enseñara sólo en mis cursos del Pedagójico las teorías de la filolojía y lingüística modernas i su aplicación pedagójica. Los profesores preparados según estos nuevos rumbos científicos encontraban resistencia constante y obstinaz entre los profesores de gramática preparados en la antigua escuela. Los reglamentos de exámenes del bachillerato, por ejemplo, mantenían las fórmulas de antaño. Era necesario hacer propaganda a favor del progreso científico en esta materia (1924: 1246).

Como el propio Lenz señala, esta "propaganda" se efectuó a través de una memoria presentada en un congreso científico y una ponencia pública en la Universidad de Chile con el sugerente nombre de Para qué estudiamos gramática. En estas instancias, junto con explicar el sentido de la reforma de 1893, defendió la necesidad de adecuar las enseñanzas gramaticales a los avances que había experimentado la ciencia lingüística en los últimos años. Como siempre, sus referentes eran europeos: "la enseñanza de la gramática en estos años está pasando por un período de crisis no sólo en Chile, sino también en Francia" (1912 [1955]: 14); y añade que hay revisión de la terminología gramatical y su aplicación escolar en Alemania e Inglaterra. El tenor de sus palabras permite aquilatar la oposición que tuvo que enfrentar en el país. Es sintomático que haya debido afirmar que sus ideas

[a]dversas a la antigua escuela gramatical (y que he profesado en el Instituto Pedagógico desde hace más de 15 años), no son herejías personales mías, sino ideas aceptadas por todos los filólogos y lingüistas modernos (p. 14).

Junto con buscar apoyo en la autoridad de otros científicos europeos (Brunot, Sweet), Lenz contextualizó los aportes de Bello, destacando no solo su "extraordinario valor científico" (p. 18), sino indicando, además, sus límites y deficiencias. La forma en que está escrito el artículo sugiere, también aquí, que Lenz estaba consciente de que pisaba terreno inseguro al atreverse a criticar al maestro venezolano. "Antes de continuar, quiero dejar bien sentado que aprecio la gramática de Bello como el que más" (p. 19), escribe. Y se preocupa de aclarar que desde hace más de tres lustros explica las ideas esbozadas por Bello y comprobadas científicamente; y que allí donde no las sigue - porque han sido rechazadas por la ciencia moderna-, el propio Bello había sugerido ya en alguna nota que él "se daba cuenta de que su teoría no estaba bien fundada". Lenz no escatima elogios para el gramático y su obra, que "significaba un gigantesco paso adelante en la numeración, denominación y explicación de los fenómenos característicos de la lengua castellana". El veneno, por cierto, está en la cola: la propia 
excelencia de la tarea de Bello "tuvo también un resultado funesto. Erigió a su autor en autoridad absoluta, infalible". En esto, por cierto, quienes defendían acríticamente las ideas del maestro, no eran fieles, según Lenz, al espíritu científico de Bello.

En otras obras, Lenz criticó diversas materias tratadas en la gramática de Bello. Así, en su artículo de 1924, La reforma de la gramática, expone "las teorías gramaticales que conviene cambiar porque Bello se equivocó, según la opinión de la mayoría de los filólogos" (p. 1253). En conformidad a la gramática de la Real Academia, que en su versión de 1917 había incorporado cambios que la aproximaban al pensamiento de Lenz, el lingüista alemán propone, por ejemplo, emplear el término "predicado" para designar al componente que, junto al sujeto, constituye la proposición, y reservar "atributo" para el adjetivo que modifica al sustantivo, y "atributo predicativo" al adjetivo o sustantivo que constituye un predicado nominal. También critica, entre otros, el tratamiento de los pronombres y su declinación en Bello; la inclusión del pronombre él en el paradigma de los artículos; la atribución de un género neutro a los infinitivos; el tratamiento de $l o$; la sustantivación del artículo, y la caracterización de la conjunción subordinante que como sustantivo neutro demostrativo. En estas críticas, y en las otras contenidas en el artículo, Lenz, junto con contrastar las proposiciones de Bello y las de la gramática académica de 1917, revisa proposiciones de Cuervo, de Menéndez Pidal y propias. El objetivo era, por una parte, convencer a su audiencia de la necesidad de incorporar estos cambios a la enseñanza de la "gramática castellana", y, por otro lado, modificar la actitud dogmática que tenían los profesores chilenos. Lenz no deseaba sustituir una autoridad por otra. Su meta era más ambiciosa. Buscaba que la enseñanza se adecuara a las teorías científicas más avanzadas de la época, aceptando el cambio que éstas traían permanentemente consigo:

Verdad científica es en cada momento la esplicación que parece la mejor HASTA AHORA, hasta que se perfeccione por nuevos progresos de la ciencia. Aceptemos los progresos! (p. 1276).

Comprometido como estaba con la instauración y el desarrollo de la lingüística científica en Chile, Lenz presionó para que oficialmente se abandonara la sujeción irrestricta a las ideas del sabio venezolano:

La cuestión grave es que el Consejo de Instrucción Pública debe resolver si la enseñanza de la gramática castellana en Chile debe seguir amarrada esclusivamente a las teorías que Andrés Bello estableció hace 75 años, $o$ si los profesores en adelante tendrán el derecho o más bien, la obligación, 
de aceptar los progresos de la ciencia filolójica que en gran parte ya están consagrados por la Real Academia de la Lengua (Lenz 1924: 1276).

Si bien Lenz no fue el único ni el primero en criticar la Gramática de Bello ${ }^{22}$, ciertamente la solidez de sus reparos y la constancia con que los divulgó deben haber sido factores fundamentales para que se lo considerara el gran contradictor de Bello. Indudablemente, la mayor contribución científica que se desprendió de esta preocupación por las cuestiones gramaticales fue su espléndido volumen La oración y sus partes, publicado en Madrid en 1920, en respuesta a una conversación sostenida por su autor con Menéndez Pidal a fines de 1914. Como el propio Lenz ha escrito, esta obra tuvo, en su origen, un objetivo práctico enmarcado en su lucha por la reforma gramatical en Chile (v. Lenz 1924).

Finalmente, la cuestión de la gramática que debía enseñarse se resolvió recién en 1935, mediante un decreto aprobatorio de los programas de estudio para la educación secundaria, en que se recomendaba a los profesores "se expongan doctrinas modernas, aceptadas por grandes filólogos e incorporadas ya, en su mayor parte, en los textos oficiales de la Academia Española" (cit. en Rojas 1940: 235). Con respecto a los verbos, se explicitaba que el profesorado debía exigir la terminología académica reformada. A juicio de Rojas, la situación generada por dicho decreto hacia 1940 era la de una enseñanza híbrida en que se mezclaban las doctrinas académicas con las de Bello.

\section{CONCLUSIÓN}

Rodolfo Lenz no solo desempeñó un papel fundamental en la introducción de la lingüística científica moderna en Chile, sino también en las transformaciones que tuvieron lugar en el modo de enseñar el idioma patrio entre fines del XIX y principios del XX. En cumplimiento de sus funciones como profesor del Instituto Pedagógico, Lenz se hizo cargo de dos problemas centrales en la enseñanza del castellano: qué variedad de lengua enseñar

22 Rojas (1940) señala que cinco años antes de la llegada de Lenz a Chile, Zorobabel Rodríguez había planteado que esta obra no podía emplearse como texto de enseñanza por su volumen, minuciosidad y exceso de clasificaciones. 
y cómo hacerlo. Con respecto al primero, se preocupó de la cuestión de la determinación de lo que hoy llamaríamos la lengua estándar y de su relación con las variedades vernáculas, desde la perspectiva de un lingüista que no solo reconocía valor a los distintos dialectos de una misma lengua histórica, particularmente los populares, sino que veía en el "lenguaje vulgar" la manifestación más genuina del espíritu del pueblo chileno. Si bien desde el punto de vista estrictamente científico no hallaba interés en el estudio de la lengua literaria ("esa lengua castiza, la que se aprende en las clases de gramática castellana, esa lengua, por el momento, no me interesa nada" (1894a: 122), llegó a escribir), reflexionó sobre la cuestión del estándar desde una posición ajena al purismo y próxima a lo que hoy consideraríamos una visión funcionalista del lenguaje.

Con respecto al segundo problema, criticó el papel que se asignaba a la gramática en la enseñanza -fruto, a su juicio, de la confusión entre ciencia y arte- y defendió un método centrado en la práctica del idioma, en que la lectura de textos modélicos tomados no solo de las artes literarias, sino del periodismo, las ciencias y la cultura en general, desempeñaba un rol medular. También censuró el empleo acrítico de la gramática de Bello y defendió la actualización teórica y conceptual en la enseñanza gramatical. En todos estos casos, se apartó de las doctrinas bellistas, que tenían fuerte ascendiente en el país. Lejos de restringirse en estas materias a la reflexión intramuros de la academia, Lenz se preocupó de difundir sus ideas entre la elite de la época con el fin de incidir políticamente en el diseño curricular y en la formación de los futuros profesores de castellano, constituyéndose en un actor relevante en la discusión y ejecución de las políticas lingüísticas en un período crítico en la historia social y cultural de Chile.

\section{REFERENCIAS BIBLIOGRÁFICAS}

Alfón, Fernando. 2011. La querella de la lengua en Argentina (1828-1928). Tesis para optar al grado de Doctor, Universidad Nacional de La Plata.

BELlo, ANDRÉs. 1833-1834 [1940]. Advertencias sobre el uso de la lengua castellana dirigidas a los padres de familia, profesores de los colegios y maestros de escuela. En VV.AA. El español en Chile. Trabajos de Rodolfo Lenz, Andrés Bello y Rodolfo Oroz. Traducción, notas y apéndices de Amado Alonso y Raimundo Lida, pp. 49-76. Buenos Aires: Biblioteca de Dialectología Hispanoamericana.

1837 [1875]. Principios de ortolojía y métrica de la lengua castellana. $3^{\mathrm{a}}$ edición. Santiago de Chile: Imprenta de la República.

1847 [1928]. Gramática de la lengua castellana destinada al uso de los americanos. Vigésima tercera edición hecha sobre la última del Autor con extensas notas y copiosos indices alfabéticos de D. Rufino José Cuervo. París: Andrés Blot, Editor. 
Blanco, Mercedes Isabel. 1994. Aspectos históricos e ideológicos de las actitudes lingüísticas en el Río de la Plata y Chile (1810-1850). En María Beatriz Fontanella de Weinberg (comp.). El español en el Nuevo Mundo: estudios sobre historia lingüística hispanoamericana, pp. 99-120. Washington, D.C. Agencia Interamericana para la Cooperación y el Desarrollo.

Campos Harriet, Fernando. 1960. Desarrollo educacional 1810-1960. Santiago de Chile: Editorial Andrés Bello.

Cartagena, Nelson. 2002. Apuntes para la historia del español en Chile. Santiago de Chile: Cuadernos de la Academia Chilena de la Lengua, Serie Estudios idiomáticos.

COOPER, ROBERT L. 1997. La planificación lingüistica y el cambio social. Cambridge-Madrid: Cambridge University Press.

Correa, Sofía, Consuelo Figueroa, Alfredo Jocelyn-Holt, Claudio Rolle y Manuel Vicuña. 2001. Historia del siglo XX chileno. Santiago de Chile: Editorial Sudamericana.

Daoust, Denise. 1997. Language planning and language reform. En Florian Coulmas (ed.). The handbook of sociolinguistics, pp. 436-452. Oxford: Blackwell.

Del Valle, José. 1999. Lenguas imaginadas: Menéndez Pidal, la lingüística hispánica y la configuración del estándar. Bulletin of Hispanic Studies 76(2): 215-233) [en línea]. Disponible en http://elies.rediris.es/elies16/Valle.html [Consulta 08/05/2016]

Ferreccio, Mario. s.f. Presentación. En Rodolfo Lenz. Diccionario etimológico de las voces chilenas derivadas de las lenguas indígenas americanas, edición dirigida por Mario Ferreccio, pp. 7-24. Santiago de Chile: Universidad de Chile.

Flores Gutiérrez, Carlos. 1950. Labor pedagógica del Dr. Rodolfo Lenz en Chile. Seminario de Didáctica General, Facultad de Filosofía y Educación, Universidad de Chile.

Gallardo, Andrés. 1982. Andrés Bello y la conciencia del idioma. Atenea 445: 123-136.

Gómez Asencio, José J. 2009. De "gramática para americanos" a "gramática de todos". El caso de Bello (1847). Revista Argentina de Historiografia Lingüistica I (1): 1-18

Góngora, Mario. 1981. Ensayo sobre la noción de estado en Chile en los siglos XIX y XX. Santiago de Chile: Ediciones La Ciudad.

Guitarte, Guillermo. 1991. Para una periodización de la historia del español de América. Siete estudios sobre el español de América. México: Universidad Nacional Autónoma de México.

Hobsbawm, Eric. 1991. Naciones y nacionalismo desde 1780. Barcelona: Crítica.

JAKSIC, IvÁn y Sol Serrano. 1990. In the service of the nation: The establishment and consolidation of the Universidad de Chile, 1842-1879. Hispanic American Historical Review 70 (1): 139-171.

JenschKe, María Luisa. 1923. Nociones de lingüistica jeneral según las clases del Prof. Dr. Rodolfo Lenz. Santiago de Chile: Imprenta Universitaria.

Lenz, Rodolfo. 1892. La fonética. Anales de la Universidad de Chile LXXXI: 901-924.

1894a. Ensayos filolójicos americanos. Anales de la Universidad de Chile LXXXVII: $113-132$ y 353-367.

1894b. Apuntaciones para un testo de ortolojía i ortografía de la lengua castellana. Anales de la Universidad de Chile LXXXVIII: 107-136.

1899. Memoria sobre las tendencias de la enseñanza del idioma patrio en Chile. Santiago de Chile: Imprenta Cervantes.

1906. Reseña de "Elementos de gramática de la lengua castellana, según las doctrinas de don Andrés Bello", por Marcelino Larrazábal Wilson. Anales de la Universidad de Chile CXIX: 40-44.

1910 [s.f.]. Diccionario etimológico de las voces chilenas derivadas de las lenguas indigenas americanas, edición dirigida por Mario Ferreccio. Santiago de Chile: Universidad de Chile. 
1912 [1955]. Para qué estudiamos gramática. Santiago de Chile: Universidad Técnica del Estado.

1918. Sobre el estudio de idiomas. Carta al señor don Julio Saavedra Molina. Anales de la Universidad de Chile CXLII: 173-264 y 289-301.

1920. La enseñanza del castellano i la reforma de la gramática. Anales de la Universidad de Chile CXLVI: 344-395.

1920 [1935]. La oración y sus partes. Estudios de gramática general y castellana. $3^{\text {a }}$ edición. Madrid: Publicaciones de la Revista de Filología Española.

1924. La reforma de la gramática. Separata de Anales de la Universidad de Chile, serie segunda, II: 1243-1276.

Parada, Ercilia. 1954. El Dr. Rodolfo Lenz y su labor pedagógica. Memoria de prueba para optar al título de profesora de Estado en la asignatura de castellano, Universidad de Chile.

Ramírez Necochea, Hernán. 1964. Panorama de la vida chilena en la época de fundación del Instituto Pedagógico. En Instituto Pedagógico. LXXV aniversario de su fundación. 1889-1964. Santiago de Chile: Universidad de Chile, Facultad de Filosofía y Educación.

Rojas Carrasco, Guillermo. 1940. Filología chilena. Guía bibliográfica y crítica. Santiago de Chile: Imp. y Lit. Universo.

SAAVEdRA, Julio. 1918. Contestación al señor don Rodolfo Lenz. Anales de la Universidad de Chile CXLII: 265-288.

Soto, Guillermo. 2015. Rodolfo Lenz: nacionalidad y lenguaje. Ponencia presentada en el Primer Congreso Latinoamericano de Glotopolítica, Santiago de Chile.

Subercaseaux, Bernardo. 1997. Historia de las ideas y de la cultura en Chile. Tomo II: Fin de siglo: La época de Balmaceda. Santiago de Chile: Editorial Universitaria. 